der Kultusminister bereits mit dem Reichskanzler in Verbindung gesetzt und eine Erweiterung des $\S 35$ der Reichsgewerbeordnung in Anlegung gebracht. Schliesslich empfiehlt Ministerialdirektor Förster als wirksamstes Mittel die Belehrung und Aufklärung des Volkes über die Verderblichkeit des Kurpfuscherthums.
J. S.

\title{
Aus dem Abgeordnetenhause.
}

Für eine energische Bekämpfung der Kurpfuscherei trat Dr. Eckels in der Sitzung des Abgeordnetenhauses vom 18. März mit allgemeinen Gründen und unter Anführung einiger drastischer Beispiele lebhaft ein. Er empfiehlt, falls der Weg der Reichsgesetzgebung nicht gangbar sein sollte, wenigstens dem gröbsten Unfug durch die Landesgesetzgebung zu stevern, und verweist in diesem Sinne auf die - auch in unserer Wochenschrift seinerzeit wiedergegebene - Hamburger Polizeiverordnung vom 1. Juli 1900, nach welcher alle Schwindelannoncen für das Hamburgische Staatsgebiet verboten sind. Die Zulässigkeit einer solchen Verordnung ist durch ein Urtheil des Reichsgerichts vom 29. Dezembel 1899 gewährleistet, worin erklärt ist, dass das allgemeine Verbot der Beförderung einer schädlichen und gefährlichen Quacksalberei insoweit Geltung beanspruchen könne, als der Quacksalbereibetrieb den Charakter eines öffentlich betriebenen Unfugs annehme. Dr. Eckels schloss seine eindrucksvollen Ausführungen mit folgenden Worten: "Nach meiner Meinung würde die beste Hilfe sein, wenn man die Wiedereinführung der Kurpfuschereiverbote erreichen könnte. Ist das nicht der Fall, so wïrde ich es für erwünscht halten, wenn die Reichsgesetzgebung im Sinne der Hamburger Verordnung vorgehen würde. Meine Herren, wenn Sie bedeuken wollen, dass unter diesem Krebsschaden, den ich eben darzulegen mil gestattet habe, gerade der ärmere Theil der Bevölkerung ausserordentlich schwer leidet, so werden Sie mir zugeben müssen, dass wir es hier auch mit einer Art von Sozialpolitik zu thun haben, und zwar mit einer nach meiner Meinung hochwichtigen“.

Zu seiner Erwiderung betonte der Direktor der Medizinalabtheilung, Wirkl. Geh. Oberregierungsrath Dr. Förster, es könne nicht geleugnet werden, dass die Kurpfuscherei in den letzten Jahren nicht nur im äusseren Umfange zugenommen habe, sondern dass auch in der Form ihres Geschäftsbetriebes eine bedauerliche Steigerung aufdringlicher Dreistigkeit und Anmaassung “ hervorgetreten sei. Ihre Bekämpfung durch Wiedereinführung eines Kurpfuschereiverbots stosse indessen auf grosse Schwierigkeiten, insbesondere da die Aussicht auf Erfolg bei den gesetzgebenden Körperschaften in dieser Richtung kaum vorhanden sei. Wir müssten uns also bescheiden, die Kurpfuscherei mit anderweitigen Mitteln zu bekämpfen. Als solche Mittel könne insbesondere in Betracht kommen einmal die Einführung einer schärferen Controlle über das Treiben der Kurpfuscher. In ihrer neuen Dienstanweisung ist den Kreisärzten zur Pflicht gemacht, auf die Kurpfuscher ihres Amtsbereichs ein wachsames Auge zu haben, Verzeichnisse über sie zu führen und darin Angaben iiber ihr Vorleben, Gesundheitsbeschädigungen, über die von den einzelnen betriebenen Spezialitäten, über ihre gerichtlichen Bestrafungen zu machen etc., anch Fälle von Gesundheitsbeschädigungen sofort dem Gericht anzuzeigen. Eine fernere Maassregel gegen die Kurpfuscher bilde die Anwendung des Reichsgesetzes über den unlauteren Wettbewerb. Endlich wäre zu erwägen, ob es sich nicht empfehle, bei den Kurpfuschern, ähnlich wie das bei einer Reihe anderer Gewerbe der Fall sei, die Möglichkeit herbeizuführen, dass ihnen wegen festgestellter Unzuverlässigkeit nachträglich der Gewerbebetrieb untersagt werde. Zur Zeit biete die Gesetzgebung hierfür keine Handhabe, a $\iota$ der bezügliche \$3 3 der Reichsgewerbeordnung sich auf Kurpfuscher nicht erstrecke. Doch habe sich 